\title{
Ocular complications in the old and glucose-intolerant genetically obese (fa/fa) rat
}

\author{
A. Dosso ${ }^{1}$, E. Rungger-Brändle ${ }^{1}$, F. Rohner-Jeanrenaud ${ }^{2}$, E. Ionescu ${ }^{2}$, C. Guillaume-Gentil ${ }^{2}$, B. Jeanrenaud ${ }^{2}$ \\ and P. M. Leuenberger ${ }^{1}$ \\ ${ }^{1}$ Laboratoire de Microscopie électronique, and ${ }^{2}$ Laboratoire de Recherches Métaboliques, Faculty of Medicine, Geneva, Switzerland
}

\begin{abstract}
Summary. Genetically obese fatty (fa/fa) male rats with abnormal oral glucose tolerance associated with initial hyperinsulinaemia as well as control lean (FA/FA) rats were investigated for the development of retinal microangiopathies. The animals were kept on a standard or sucrose supplemented diet. When tested at 60 weeks, the glucose intolerance of fa/fa rats was accompanied by an insulin response that was now either comparable to that of lean rats (standard diet) or close to nil (sucrose supplemented diet). At killing (68 weeks of age), retinal vasculature was examined by electron microscopy and morphological changes were quantitatively assessed by ultrastructural morphometry. A retinal microangiopathy was observed in all mutant animals which was more pronounced in the sucrose fed group, and which was characterized by: (1), an increase in focal thickenings and in nodules
\end{abstract}

of the basement membrane adjacent to the perivascular glial cells; (2), a decrease in the number of pericyte nulei with concomitant signs of early degenerative cytoplasmic changes of pericytes; (3), an increase in the pinocytic activity of endothelial cells, indicative of presumptive changes in vascular permeability; (4), an increase in the number of intercellular endothelial junctions; (5), the presence of numerous stimulated platelets within capillaries. The fa/fa rat may thus be considered as a suitable model for studying the pathophysiology of ocular complications, in particular retinopathy accompanying non-insulin-dependent diabetes.

Key words: Obese fa/fa rat, non-insulin-dependent diabetes, oral glucose tolerance, diabetic microangiopathy, retinal capillary.
By analogy to non-insulin-dependent diabetes mellitus in humans, an animal model of this syndrome ought to present a genetic predisposition for diabetes and should be obese, hyperglycaemic, glucose intolerant, and initially, hyperinsulinaemic [1]. Although a number of rodents and primates with non-insulin-dependent diabetes mellitus are known [1-4], they have not, except in a few instances [4], been studied to unravel long-term complications such as peripheral neuropathy, nephropathy and retinopathy.

The fatty ( $\mathrm{fa} / \mathrm{fa}$ ) rat first described by Zucker [5] is only slightly hyperglycaemic in the basal state $[6,7]$. However, its oral glucose tolerance is known to be impaired, an abnormality that worsens with time (more marked at 1314 weeks than at 6-7 weeks) [6]. Concomitantly with this abnormal oral glucose tolerance, the fa/fa rat markedly hypersecretes insulin before or following glucose ingestion, i. e. about ten times more than that of control rats [6]. Nevertheless, it is not known how the oral glucose tolerance and the pattern of the insulin response evolve with age in fa/fa rats, nor it is known whether this animal develops diabetic complications. The aim of the present study was, therefore, to perform oral glucose tolerance tests in old fa/fa rats, as well as to study the potential development of degenerative changes in the retinal microvasculature. A potential aggravating influence of a high sucrose diet on these parameters was also tested $[8,9]$.

\section{Materials and methods}

\section{Animals}

Homozygous male fatty (fa/fa) rats and their lean controls (FA/FA), bred in an animal quarter with a constant temperature $\left(22^{\circ} \mathrm{C}\right)$ and a fixed (12-h) artificial light cycle were used. They had free access to a standard laboratory chow (UAR, Epinay, Villemoisson, France) and to either water or to a $10 \%$ sucrose solution from 5 weeks of age up to 68 weeks. Initially, 24 rats were divided into 4 groups of 6 rats (lean or obese; standard diet + water or standard diet + sucrose). Two obese sucrose-fed animals died prior to the oral glucose tolerance test (OGTT) performed at 60 weeks. One lean and two obese rats fed the standard diet alone died after the OGTT as well as one more obese sucrose-fed. The remainders (11 FA/FA and $7 \mathrm{fa} / \mathrm{fa})$ were killed at 68 weeks of age.

It should be mentioned that,-although no autopsy was performed at death, all obese rats (sucrose-fed or not) had become albuminuric with age, as assessed by using Combur strips (Combur 9-test, Boehringer, Mannheim, FRG). 


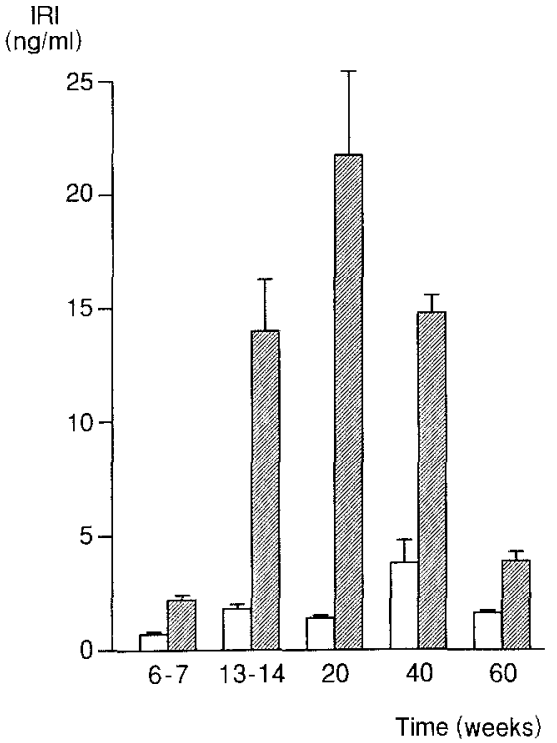

Fig. 1. Basal insulinaemia of unanaesthetized lean (FA/FA) $\square$ and genetically obese (fa/fa) rats as a function of age. Mean \pm SEM of six experiments per group. Statistical significance lean vs obese, $p$ always $<0.001$. IRI $=$ plasma insulin concentration

\section{In vivo measurements}

Blood samples were collected at $0^{\circ} \mathrm{C}$ by tail clipping. Basal insulinaemia of different groups of unanaesthetized lean and obese rats fed the standard diet and water only was measured at 6-7, 13-14, 20, 40 and 60 weeks of age. An OGTT (spontaneous ingestion of $1 \mathrm{~g} / \mathrm{kg}$ lean body weight by unanaesthetized animals) was performed at the age of 60 weeks according to a method described previously [6]. Blood samples were collected at -10 and 0 min for basal values of glycaemia and insulinaemia and at 5, 15,30,60, 120, 180 and 240 min after the glucose load.
Plasma glucose levels were measured by the glucose oxidase method [10], while plasma insulin concentrations were radioimmunoassayed with dextran-coated charcoal separation of the bound and free fractions [11].

\section{Electron microscopy}

Rats were anaesthetized with ether, enucleated and killed by an overdose of ether. After enucleation, one eye per animal was processed for electron microscopy by fixation in $2.5 \%$ glutaraldehyde, $0.1 \mathrm{~mol} / \mathrm{l}$ cacodylate buffer ( $\mathrm{pH} 7.0), 0.05 \%$ tannic acid (Mallinckrodt Inc., Paris, Ky, USA). Eyes were opened at the ora serrata, immersed immediately into cold fixative and kept on ice for $30-45 \mathrm{~min}$; then, they were cut into small pieces and fixed in the same fixative for six additional h at $4^{\circ} \mathrm{C}$. Tissue pieces were washed overnight in several changes of $0.1 \mathrm{~mol} / \mathrm{l}$ cacodylate buffer $(\mathrm{pH} 7.4)$, postfixed with $1 \%$ osmium tetroxide $\left(1 \mathrm{~h}\right.$ at $\left.4^{\circ} \mathrm{C}\right)$, dehydrated through a graded series of ethanol and embedded in Spurr. The other eye was quickfrozen in liquid nitrogen and stored at $-70^{\circ} \mathrm{C}$ for further studies.

Semi-thin sections were stained with toluidine blue. Ultra-thin sections were mounted on 200 mesh palladium-coated copper grids, stained with uranyl acetate and lead citrate [12], and viewed with a Philips EM 300 electron microscope. Photographs were taken on a fine grain release positive $36 \mathrm{~mm}$ roll film (Kodak-Eastmann film, no. 5302) at a magnification of $4900 \times$. In order to standardize magnification between individual films, calibration grids were photographed at the start of every new film each containing 30 exposures.

\section{Morphometry of the retinal capillaries}

Based on a previous study [13] showing structural differences of the vessels between the individual layers of the retina, we limited our studies to the outer plexiform layer, where half of the retinal capillaries are located. Between three and six retinae per experimental group corresponding to the number of surviving animals, were examined. Six to ten capillaries were photographed randomly from

Table 1. Morphometry on capillary circumference and basement membrane in lean (FA/FA) and obese (fa/fa) rats

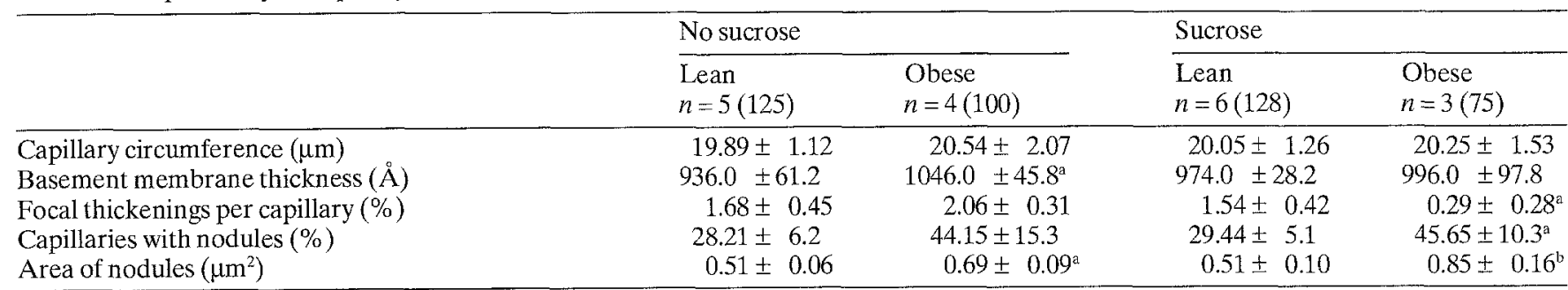

The data were obtained by measuring or counting a total of 428 cross-sectioned capillaries of the outer plexiform layer. The values indicate the mean \pm SD. $n$ corresponds to the number of animals investigated. The numbers in parentheses represent the number of capillaries examined in each experimental group. The significance of difference was assessed by the Student's $t$-test ( ${ }^{\mathrm{a}} p<0.05 ;{ }^{\mathrm{b}} p<0.01$ )

Table 2. Morphometry on endothelial cells, pericytes, and platelets

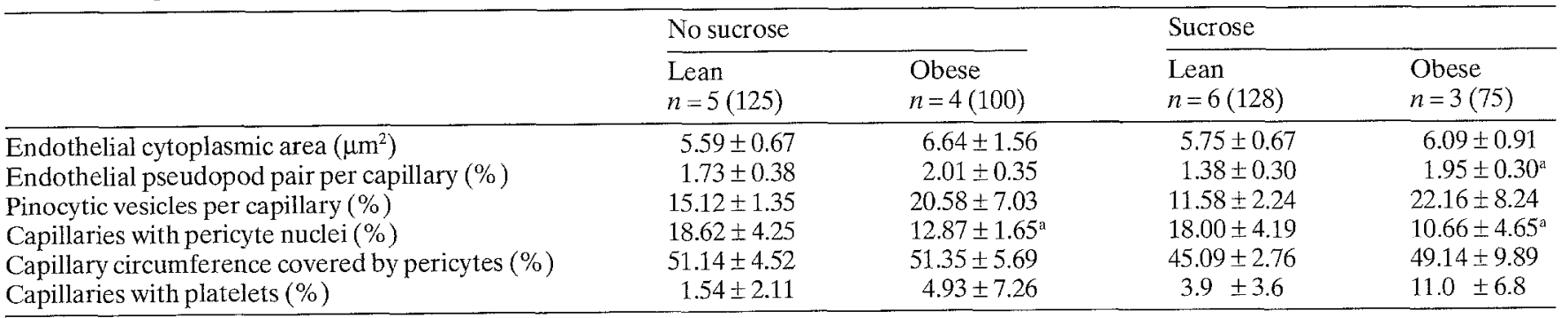

For symbols and explanations, see Table 1 
four different tissue blocks of each retina. Thus, a total of 428 capillaries were taken for morphometric evaluation.

After conversion to positive film, the morphometric analysis was done on a light screen at a final magnification of $17000 \times$. A QT computer was used for the calculation of the following parameters: (1) capillary circumference, measured as the total length of the abluminal endothelial cell membrane, (2) endothelial cytoplasmic area, measured as the total endothelial surface delineated by the outer circumference minus the luminal and the nuclear surfaces. (3) portion of the capillary circumference covered by cytoplasmic processes of the pericyte, expressed as the percentage of total capillary circumference, (4) mean area of extracellular nodules, defined as the area of basement membrane material surrounding collagenous fibrils. Moreover, we counted the number of pericyte processes per capillary, basement membrane nodules, focal thickenings of the basement membrane, endothelial pinocytic vesicles, cytoplasmic processes at intercellular endothelial junctions, pericyte nuclei, and platelets (Fig. 3, 4 and 5).

The width of the capillary basement membrane was measured by the two-point minimum method [14] which avoids overestimation and which has proved to be accurate for measuring retinal capillary basement membrane thickness [15]. All measurements were carried out without prior knowledge of capillary origin.

\section{Statistical analysis}

Mean and SD were determined for each parameter, $n$ representing the number of animals examined. The significance of differences was assessed by the Student's $i$-test.

\section{Results}

\section{In vivo measurements}

Figure 1 shows the evolution of basal plasma insulin levels in lean and genetically obese fa/fa rats as a function of time. Basal insulinaemia on lean rats remains approximately constant from 6-7 weeks up to 60 weeks. That of obese animals varies with time. It progressively increases to reach a peak at 20 weeks, to then decrease toward values measured at the young age. Figure 2 shows the results of an OGTT performed on 60-week-old lean and obese rats maintained on either the standard diet with water (left panels) or on the same diet with a $10 \%$ sucrose solution (right panels). All obese rats present an abnormal glucose tolerance compared to that of their respective controls, the abnormality being exaggerated by sucrose feeding. In response to the glucose challenge, insulinaemia of the obese group without sucrose, only doubles (compared to the basal levels), as it does in the two groups of lean controls. Moreover, plasma insulin levels of the obese group chronically fed with sucrose barely increased following the ingestion of the glucose load.

\section{Intraretinal capillaries}

General features. In the material that had been fixed by immersion, the circumference of capillaries within the outer plexiform layer measures about $20 \mu \mathrm{m}$ in all four experimental groups (for exact values of the measured pa-
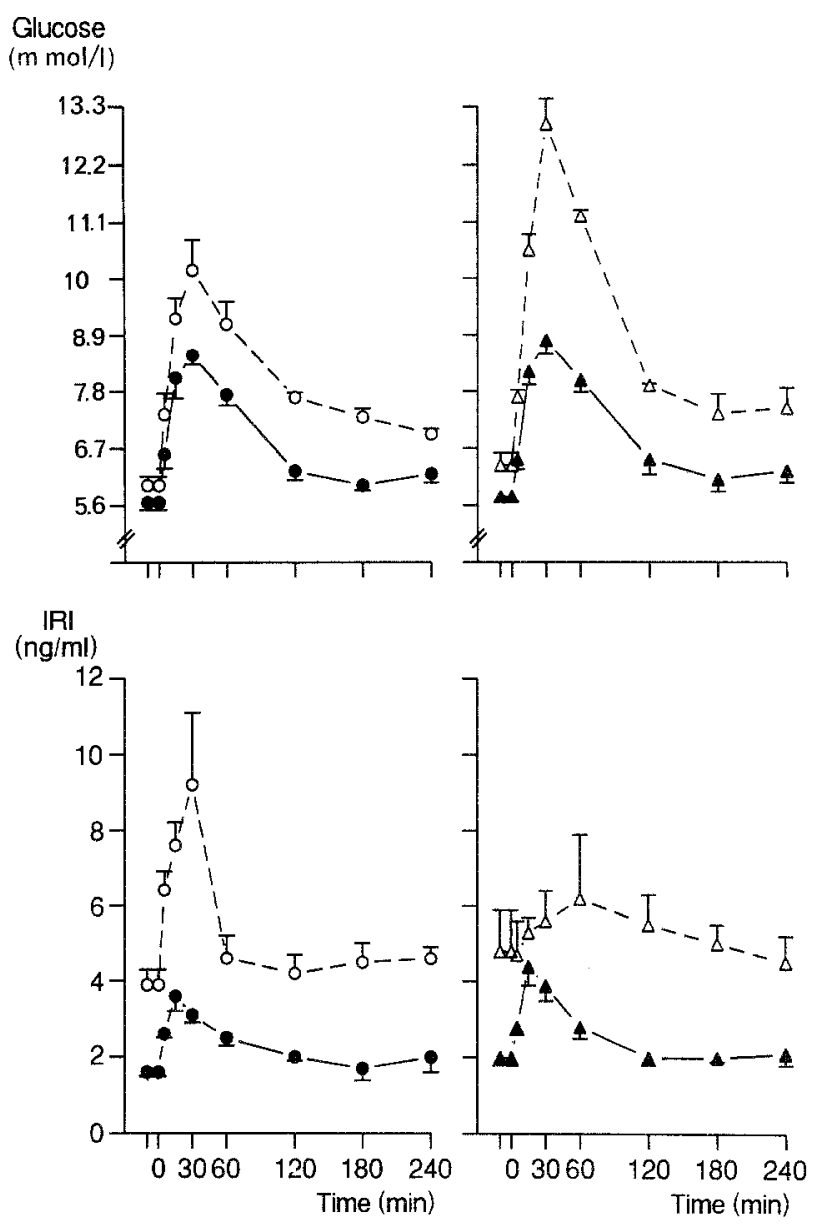

Fig. 2. Plasma glucose and insulin levels of unanaesthetized 60week-old lean (FA/FA) and genetically obese ( $\mathrm{fa} / \mathrm{fa}$ ) rats before and after the spontaneous ingestion (at time 0 ) of $1 \mathrm{~g} / \mathrm{kg}$ lean body weight glucose. The animals were fed the standard diet with either water (left panels) $\bullet=$ lean, $O=$ obese or with a $10 \%$ sucrose solution (right panels) $\boldsymbol{\Delta}$ = lean, $\Delta=$ obese. Mean \pm SEM of four to six experiments per group. Left panels: obese significantly different from lean at times $30,60,120,180,240 \mathrm{~min}$ (glycaemia); at all time points (insulinaemia). Right panels: obese significantly different from lean at all time points (glycaemia); at 10, 0, 120, 180 and $240 \mathrm{~min}$ (insulinaemia). $\mathrm{IRI}=$ plasma insulin concentration

rameters see Table 1). The cytoplasmic area of the endothelial cells is slightly larger in the mutant than in the lean control, but the difference is not striking (Table 2). Thus, the capillary size, as defined by its circumference and by the cytoplasmic endothelial area, is similar in mutant and control animals. Moreover, we could not observe any clear-cut influence of sucrose diet on these two parameters in either group.

However, the width of the endothelial basement membrane is significantly larger in the obese, standard diet fed group than in the lean control $(p=0.025)$. This difference is not significant $(p=0.7)$ in those groups that had been fed on sucrose (Table 1).

Frequently, focal thickenings of the basement membrane lining the perivascular glial cells are enlarged to form nodules containing fibrillar material (Fig. $3 \mathrm{~b}$ and 5 a). The continuity between such fibrillar material and collagen fibers suggests a collagenous nature of the fibrils (Fig.5a). With the fixation method used, collagen fibers 

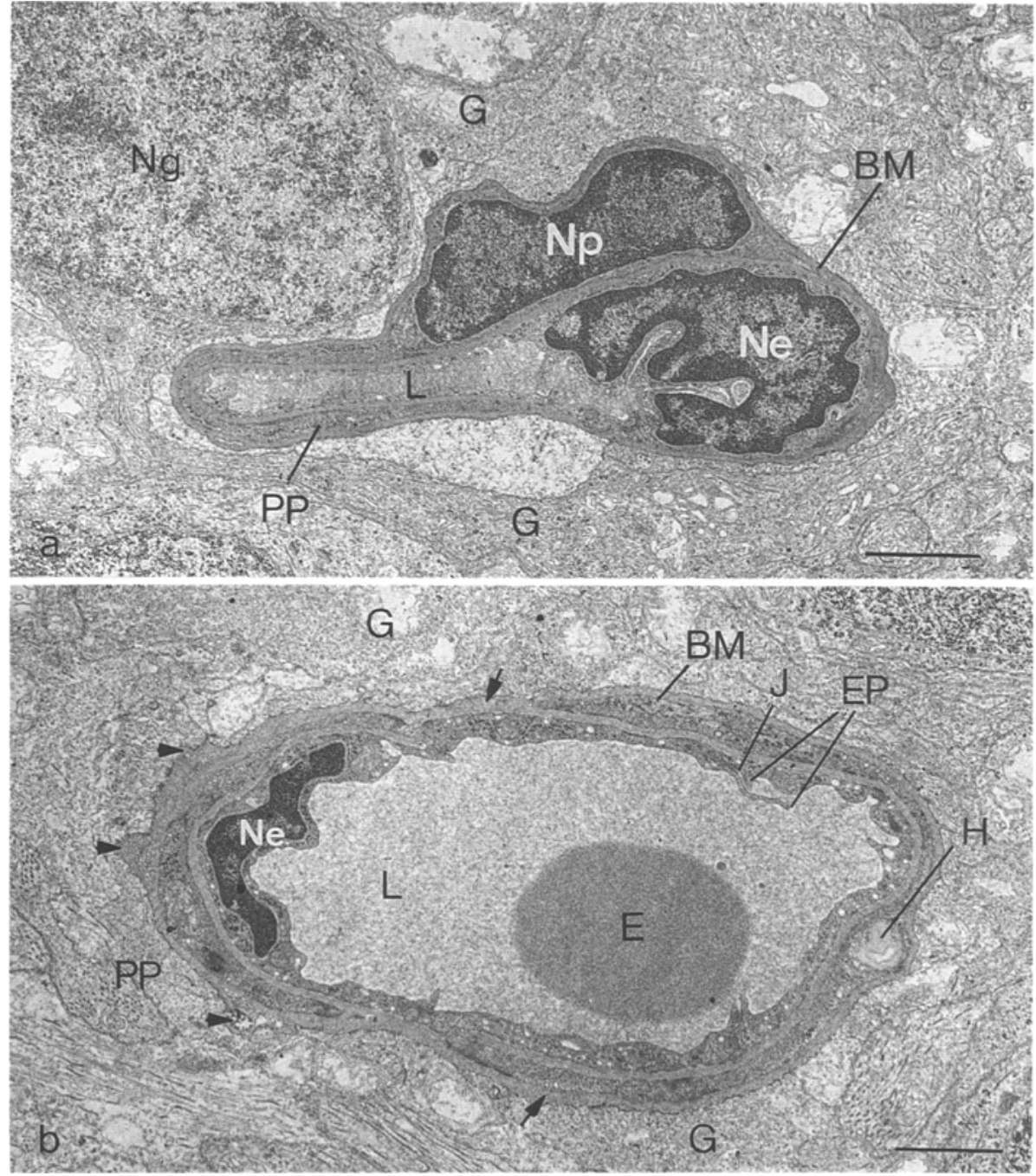

Fig. 3. Overview of capillaries from the outer plexiform layer of lean (a) and obese fa/fa (b) rats. In the mutant (b), focal thickenings (arrows) and nodules (arrowheads) of the basement membrane (BM) are observed lining perivascular glial cells $(G)$. Nodules contain fibrils and sometimes electron dense, heterogenous material. In continuity with intercellular junctions (J), endothelial cytoplasm forms pseudopodia (EP). Ne: endothelial nucleus; $\mathrm{Np}$ : pericyte nucleus; $\mathrm{Ng}$ : glial nucleus; L: lumen; PP: pericyte cytoplasmic process; E: erythrocyte; $\mathrm{H}$ : hyaline body. Bars, $1 \mu \mathrm{m}$ typically show a periodicity of $70 \mathrm{~nm}$. In the obese mutant, the number of focal thickenings per capillary is increased, especially in rats fed on sucrose $(p=0.05$, Table 1$)$. One or more nodules are found in about $29 \%$ of the control and in $45 \%$ of the mutant capillaries $(p=0.04)$. The area of these nodules is significantly larger in both obese groups, fed either on standard $(p=0.02)$ or on sucrose supplemented $(p=0.01)$ diet (Table 1$)$.

Endothelial cells. As mentioned above, the endothelial cytoplasmic area is only slightly increased in the mutant. Also, there is no obvious sign of structural alterations and the cells do not appear to be swollen, as has been described in the spontaneously diabetic BB [16] and the streptozotocin (SZ)-induced diabetic rat [17].

Intercellular junctions between endothelial cells are always flanked by pseudopod-like processes (Fig. $3 \mathrm{~b}$ and $4 \mathrm{a}$ ). They may be quite prominent and extend into the lumen of the capillary. Since they are easily visible, we have taken the number of pseudopod pairs as a measure of the number of intercellular junctions. This figure is often used to estimate the endothelial cell number [17]. The number of pseudopods per capillary is larger in obese mutants than in lean control animals (Table 2). The dif- ference is statistically significant in those experimental groups that were fed on sucrose $(p=0.03)$. Accordingly, the number of endothelial cells appears to be increased in sucrose fed obese rats.

Moreover, we have noted an increased pinocytic activity in endothelial cells of obese animals by counting the number of closed pinocytic vesicles per capillary of a size between 500 and $1000 \AA$ [18] (Fig. 4 a). In sucrose fed animals, the difference between mutant and control is even more pronounced (Table 2).

Pericytes. We have estimated the frequency of pericytes in these intraretinal capillaries by determining the percentage of capillaries with a cross-sectioned pericyte nucleus. As shown in Table 2, the percentage of capillaries with a pericyte nucleus is diminished in the obese mutant. The difference is statistically significant in both sucrose fed $(p=0.05)$ and standard fed $(p=0.05)$ animals. Nevertheless, in both mutant and control animals, the capillary circumference covered by pericytes is about $50 \%$ of the total length (Table 2). In the obese rat, masses of glycogen granules are observed within the pericytic cytoplasm (Fig. 5 b), together with hyaline bodies (Fig. 3b) and poorly defined waste products (Fig. $5 \mathrm{c}$ ). 


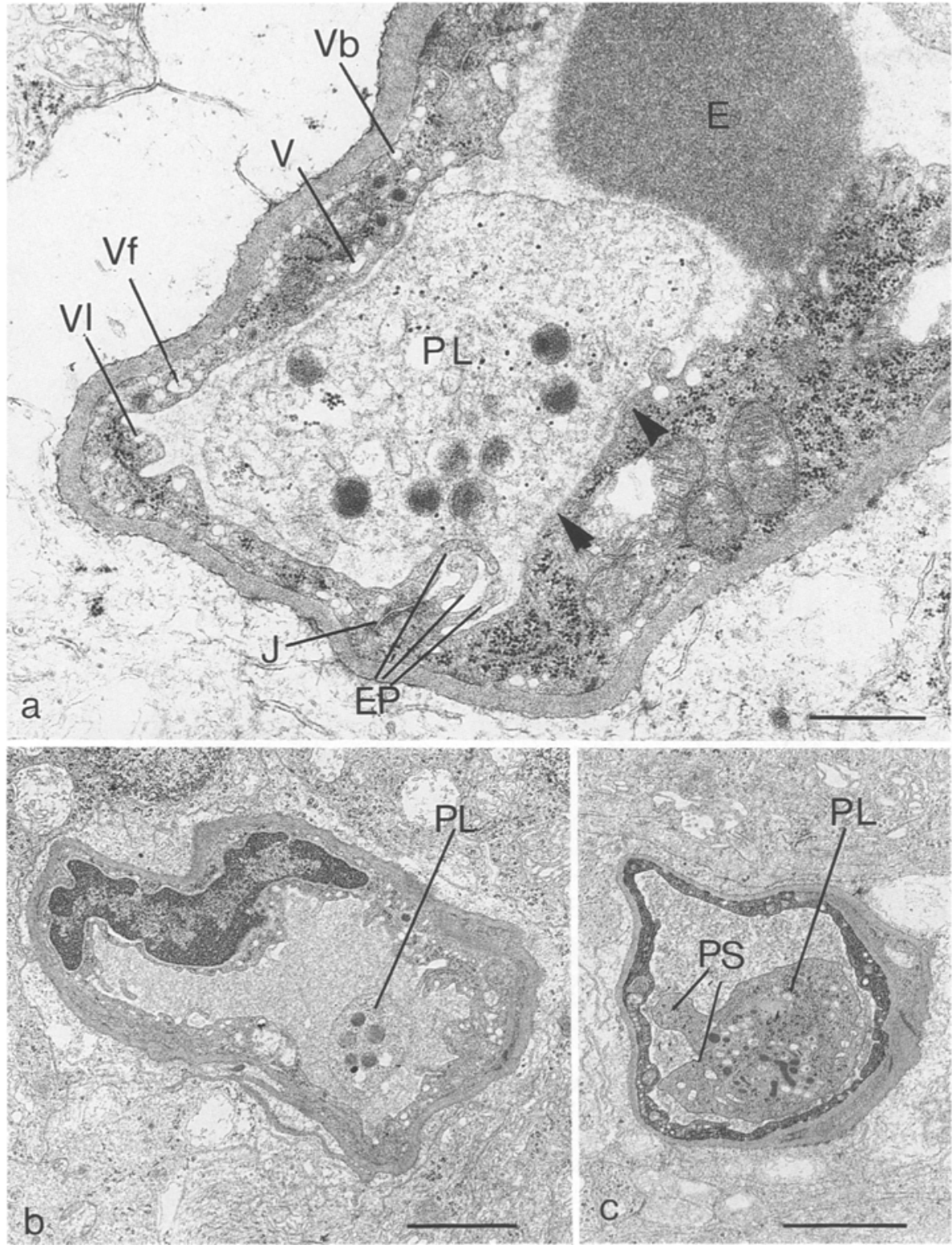

Fig.4a-c. Retinal capillaries containing platelets in their lumen (a). High magnification of a capillary of an obese $\mathrm{fa} / \mathrm{fa}$ rat with numerous pinocytic vesicles (V). Some of these vesicles are confluent (V1), others are fused with either the plasma membrane lining the basement membrane (Vb) or the vessel lumen (V1). The lumen is almost obstructed by a platelet (PL) and an erythrocyte (E); the platelet appears to adhere to the endothelial wall (arrowheads). EP: pseudopod-like processes near intercellular junction $(\mathrm{J})$. b Platelets in lean control animals typically are discoid. c Whereas in obese fa/fa rats, they display pseudopods (PS). Bars $0.5 \mu \mathrm{m}$ (a), $2 \mu \mathrm{m}$ (b), $1 \mu \mathrm{m}(\mathbf{c})$
Platelets. During this study, we were struck by the shape and the increased number of circulating platelets in intraretinal capillaries of the obese rats. In control rats, the few platelets observed were discoid, indicating resting cellular activity (Fig. 4b), whereas platelets in obese rats not only displayed numerous pseudopods (Fig. 4c) typical for an activated state, but often adhered to the endothelial wall (Fig. 4a). Also, the frequency of capillaries containing platelets was 2 to 2.5 times higher in the obese rats. In sucrose fed animals, this increase was even more pronounced (Table 2).

\section{Discussion}

The fatty (fa/fa) rat is glucose intolerant in the absence of a marked basal hyperglycaemia [6,7]. Its insulin response to glucose is markedly increased compared to that of the lean rat when tested at 6-7 weeks or at 13-14 weeks [6]. This study shows that basal insulinaemia of obese fa/fa rats decreases with age from 20 weeks onward. It also shows that, at 60 weeks, the magnitude of the glucose-induced insulin output is similar in lean and obese animals. These results suggest the occurrence, in the obese group, of a deterioration of the insulin secretion capacity. They are in keeping with morphological observations showing that the hypertrophied Beta cells of 5-week-old fa/fa rats become degranulated and fibrotic at 52 weeks [19].

A sucrose supplementation to the diet has no effect in lean rats, while it makes the obese rats hyperglycaemic in the basal state, as well as more glucose intolerant following glucose ingestion. The glucose-induced insulin output of sucrose-fed obese animals is close to nil. Thus, longterm sucrose feeding seems to be able to bring the obese $\mathrm{fa} / \mathrm{fa}$ rat from a state of glucose intolerance to another that is close to non-insulin-dependent diabetes.

Whilst it is a well established fact that insulin-dependent diabetic patients develop their retinopathy only after 

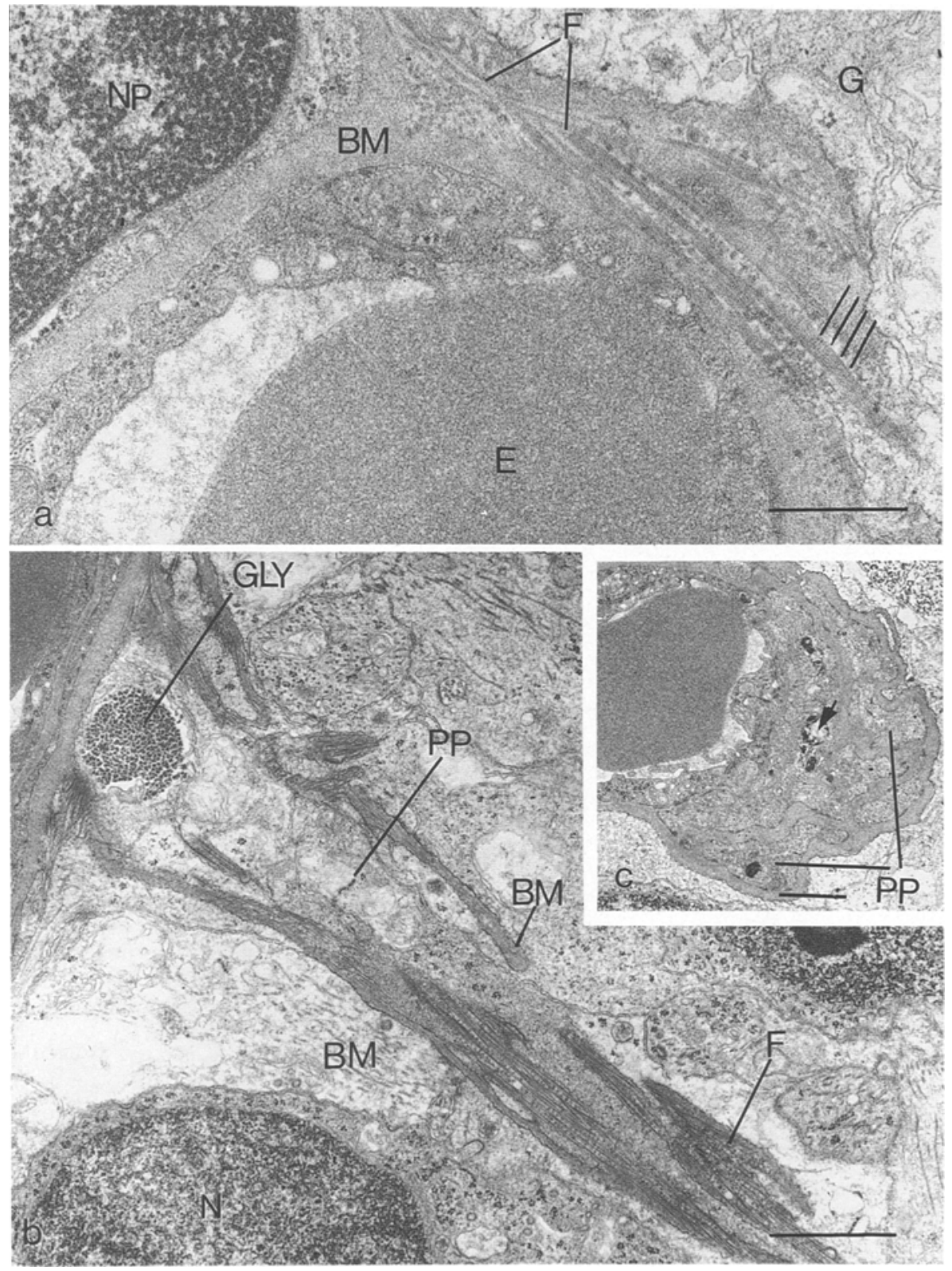

Fig. 5a-c. High magnification of capillary walls. a Nodules of the basement membrane (BM) in an obese fa/fa rat filled with fibrils which are in continuity with collagen fibers indicating the collagenous nature of the fibrils. Parallel bars denote $70 \mathrm{~nm}$ periodicity of collagen fibers. Np: pericyte nucleus. G: glial cell. E: erythrocyte. b Cytoplasmic pericyte processes $(\mathrm{PP})$ contain a massive accumulation of glycogen (GLY), and vesicular residual bodies (c, arrow). F: fibrillar material within basement membrane (BM); Ng: glial nucleus. Bars, $0.2 \mu \mathrm{m}(\mathbf{a}), 0.6 \mu \mathrm{m}(\mathbf{b}), 3 \mu \mathrm{m}(\mathbf{c})$ many years or even decades of hyperglycaemia, non-insulin-dependent diabetic patients may present retinopathy already at the time of diagnosis of their metabolic disorders. The question thus arises, whether mere glucose intolerance over years without overt hyperglycaemia may be sufficient to induce microvascular retinal changes. For obvious reasons, this question might be easier to answer if there were an animal model for non-insulin-dependent diabetes mellitus at hand, developing microvascular lesions comparable to those in humans.

Retinopathies in laboratory rodents, in particular rats with induced diabetes have been known about for over 20 years and may serve for comparison with lesions observed in obese fa/fa rats. Two insulin-dependent diabetic rodent models have been extensively studied so far, namely the spontaneously diabetic BB rat and the SZ-induced diabetic rat. However, some discrepancies have been noted by different investigators $[17,20-24]$ regarding reti- nal microangiopathies which appear to vary with the genetic background and the age of the animals at investigation.

We have compared the ocular lesions observed in our $\mathrm{fa} / \mathrm{fa}$ rats with those known from studies on insulin-dependent diabetes mellitus in rats. All mutant fa/fa rats developed cataracts that began to appear at the age of 32 weeks, later than in SZ diabetic rats [21]. The general picture of the retinal microangiopathy in the fa/fa rat is quite similar to that described for the $\mathrm{BB}[16,25]$ and the SZ $[17,20,21]$ rat although, at first sight, the alterations are less striking. Nevertheless, our morphometric analysis shows that subtle alterations such as a shift in the relative frequency of endothelial cells and pericytes as well as morphological changes in the basement membrane can be ascribed to diabetic disorders.

We have taken the number of pseudopod-like endothelial processes flanking intercellular junctions as a 
means of evaluating the number of intercellular junctions and have found an increase in the obese fa/fa rat. Since an increase in the number of junctions observed in the SZ diabetic rat has been interpreted as evidence for endothelial cell proliferation [17], it is - by analogy - conceivable, that in the fa/fa rat, the endothelial cell number is also increased. In our study, we have observed a reduced number of pericyte nuclei and an accumulation of glycogen and cellular waste products within the pericyte cytoplasm. These observations may be considered indicative of early degenerative changes in pericytes of the fa/fa rat. In the obese rat, there is no indication for capillary dilatation that would be reflected by an increase in both vessel circumference and endothelial cytoplasmic area.

Another characteristic feature of diabetic microangiopathy is thickening of the basement membrane, probably due to an increase in the basement membrane components collagen IV and laminin to compensate for the reduced synthesis of heparan sulfate proteoglycan [26, 27]. In the fa/fa rat, we did not see a prominent general increase in basement membrane width, but we observed a significant increase in the number and size of focal thickenings and nodules. Similar nodules have been observed in the SZ diabetic rat [21], in BB rats [25] and in diabetic humans $[28,29]$. They appear to occur also in senile, non-diabetic rats $[30,31]$ and in rats and dogs that have been fed on a high galactose diet $[32,33]$. In all these groups as well as in our fa/fa rats, local basement membrane thickening was accompanied by collagen fiber deposits. The mechanism of such collagen accumulation is not yet clear, but aldose-reductase activity may play some role, since it is known that collagen deposition can be prevented by administration of an inhibitor of this enzyme $[32,34,35]$. Interestingly, we found focal thickenings and nodules only in basement membrane segments lining perivascular glial cells. It is conceivable that, besides pericytes, glial cells contribute to basement membrane alterations.

Increased permeability of the blood-retinal barrier, described in diabetic humans and animals [36-39], can be the consequence not only of increased permeability of the basement membrane to blood proteins, but also of an increased pinocytic activity of the endothelial cells. Similar to the situation in SZ rats [40], fa/fa rats have an increased number of pinocytic vesicles. This suggests a very active transendothelial transport that may well contribute to modifications of the blood-retinal barrier.

Other capillary complications such as microthrombi and obliterated capillaries as they were described for the $\mathrm{BB}$ [16] and SZ rat [20], have not been observed in the fa/fa rat. However, all platelets in the mutant were activated, as judged by the abundance of pseudopods and by their adherence to the endothelial wall. This situation is likely to contribute to the development of focal ischaemia in the retina which in turn could lead to a stimulation of proliferative events.

In general, sucrose diet tends to aggravate both cataract development and retinal microangiopathy in the obese fa/fa rat. This is also the case in the SZ diabetic rat [20], where it has been possible to accelerate the forma- tion of vascular lesions, originally induced by the diabetic state, by superimposing an additional metabolic factor.

Based on our observations, we consider the obese fa/fa rat as a promising model for the study of non-insulindependent diabetic retinal microangiopathy. To the best of our knowledge, this is the first report on microvascular changes, comparable to insulin-dependent diabetic alterations, in a non-insulin-dependent diabetic animal. Here, they are apparently induced by mere glucose intolerance in the absence of overt hyperglycaemia in the fasting state. In addition, this model offers the opportunity to test the influence of nutritional factors on the severity and/or progression of microvascular lesions.

Acknowledgements. We thank Ms. B. Baumgartner for technical assistance and Ms. S. Wagner and Mr. N. Gerber for photographic work. The work has been supported by the Swiss National Science Foundation, grants no. 3.883-0.85, 3.857-0.88, and 3.822-0.86 and by a grant-in-aid of Nestlé S.A. (Vevey), Switzerland.

\section{References}

1. Howard CF (1985) Atherosclerosis and insulin in primates with diabetes mellitus. Metabolism 34 [Supp 1]: 60-69

2. Hahn von Dorsche H, Scheven C, Stiller KJ, Timm D (1984) Quantitativ-histochemische Untersuchungen an Langerhansschen Inseln von Sandratten (Psammomys obesus) während der Ausbildung eines diabetes mellitus. 1. Cytophotometrische Messung des Insulingehalts. Acta Histochem 75: 199-203

3. Hummel KP, Dickie MM, Coleman DL (1966) Diabetes a new mutation in the mouse. Science 153:1127-1128

4. Midena E, Segato T, Radin S, di Giorgio G, Meneghini F, Piermarocchis S, Belloni AS (1989) Studies on the retina of the diabetic ob/ob mouse. I Endothelial cell-pericyte ratio. Ophthalmic Res 21: 106-111

5. Zucker LM, Zucker TF (1961) Fatty, a new mutation in the rat. J Hered 52: 275-278

6. Ionescu E, Sauter JF, Jeanrenaud B (1985) Abnormal oral glucose tolerance in genetically obese (fa/fa) rats. Am J Physiol 248: E500-E506

7. Rohner-Jeanrenaud F, Proietto J, Ionescu E, Jeanrenaud B (1986) Mechanism of abnormal oral glucose tolerance of genetically obese fa/fa rats. Diabetes 35: 1350-1355

8. Cohen AM, Michaelson IC, Yanko MD (1972) Retinopathy in rats with disturbed carbohydrate metabolism following a high sucrose diet. 1. vascular changes. Am J Ophthalmol 73: 863-869

9. Yanko L, Michaelson C, Cohen AM (1972) The retinopathy of sucrose fed rats. Israel J Med Sci 73: 1632-1636

10. Bergmeyer HU, Bernt E (1963) D-Glucose. In: Bergmeyer HU (ed) Methods of enzymatic analysis. Academic Press, New York, pp 123-130

11. Herbert V, Lau KS, Gottlieb CW, Bleicher SJ (1965) Coated charcoal immunoassay of insulin. J Clin Endocrinol Metab 25: 1375-1384

12. Reynolds ES (1963) The use of lead citrate at high $\mathrm{pH}$ as an electron-opaque stain in electron microscopy. J Cell Biol 17: 208

13. Sosula L, Beaumont P, Jonson KM, Hollows FC (1972) Quantitative ultrastructure of capillaries in the rat retina. Invest Ophthalmol 11: 916-925

14. Williamson JR, Vogler NJ, Kilo C (1969) Estimation of vascular basement membrane thickness. Diabetes 18:567-578

15. Beauchemin ML, Antille G, Leuenberger PM (1975) Capillary basement membrane thickness: a comparison of two morphometric methods for its estimation. Microvas Res 10: 76-82

16. Sima AF, Chrakrabarti S, Garcia-Salinas R, Basu PK (1985) The $\mathrm{BB}$ rat - an authentic model of human diabetic retinopathy. Curr Eye Res 4: 1087-1092 
17. Sosula L, Beaumont P, Hollows FC, Jonson KM (1972) Dilatation and endothelial proliferation of retinal capillaries in streptozotocin-diabetic rats: quantitative electron microscopy. Invest Ophthalmol 11:926-935

18. Palade GE (1961) Blood capillaries of the heart and other organs. Circulation 24: 368-384

19. Shino A, Matsuo T, Iwatsuka H, Suzuoki Z (1973) Structural changes of pancreatic islets in genetically obese rats. Diabetologia 9: 413-421

20. Papachristodoulou D, Heath $\mathrm{H}$ (1977) Ultrastructural alterations during the development of retinopathy in sucrose-fed and streptozotocin-diabetic rats. Exp Eye Res 25: 371-384

21. Leuenberger PM, Cameron D, Stauffacher W, Renold AE, Babel J (1971) Ocular lesions in rats rendered chronically diabetic with streptozotocin. Ophthalmol Res 2:189-204

22. Tilton RG, LaRose LS, Kilo C, Williamson JR (1986) Absence of degenerative changes in retinal and uveal capillary pericytes in diabetic rats. Invest Ophthalmol Vis Sci 27: 716-721

23. Agrawal P, Agrawal L, Tandon H (1966) Experimental diabetic retinopathy in albino rats. Orient Arch Ophthalmol 4: 68

24. Von Sallman L, Grimes P (1972) Histopathologic changes in the eyes and kidneys of rats with long term streptozotocin diabetes. In: Bellows $\mathbf{J}$ (ed) Contemporary ophthalmology. Williams and Wilkins, Baltimore, pp 502-511

25. Chakrabarti S, Sima AF (1989) Effect of aldose reductase inhibition and insulin treatment on retinal capillary basement membrane thickening in BB rats. Diabetes 38: 1181-1186

26. Rohrbach DH, Martin GR (1982) Structure of basement membrane in normal and diabetic tissue. Ann NY Acad Sci 401:203211

27. Essner E, Lin WL (1988) Immunocytochemical localization of laminin, type IV collagen and fibronectin in rat retinal vessels. Exp Eye Res 47:317-327

28. Bloodworth JMB, Molitor DL (1965) Ultrastructural aspects of human and canine retinopathy. Invest Ophthalmol 4: 1037-1048

29. Ashton N (1974) Vascular basement membrane changes in diabetic retinopathy. Br J Ophthalmol 58: 344-363

30. Leuenberger PM (1973) Ultrastructure of the ageing retinal vascular system with special reference to qualitative and quantitative changes of capillary basal membrane. Gerontologia (Basel) 19: $1-15$
31. Nagata M, Katz ML, Robison WG (1986) Age-related thickening of retinal capillary basement membranes. Invest Ophthalmol Vis Sci 27: 437-440

32. Frank RN, Keirn RJ, Kennedy A, Frank KW (1983) Galactoseinduced retinal capillary basement membrane thickening: prevention by sorbinil. Invest Ophthalmol Vis Sci 24: 1519-1524

33. Engermann RL, Kern TS (1984) Experimental galactosemia produces diabetic-like retinopathy. Diabetes 33: 97-100

34. Robison WG, Kador PF, Kinoshita JH (1983) Retinal capillaries basement membrane thickening by galactosemia prevented with aldose-reductase inhibitors. Science 221: 1177-1179

35. Nagata M, Robison WG (1987) Aldose reductase, diabetes and thickening of the retinal inner limiting membrane. Invest Ophthalmol Vis Sci 28: 1867-1869

36. Cunha-Vaz J, Faria de Abreu JR, Campos AJ, Figo GM (1975) Early breakdown of the blood-retinal barrier on diabetes. Br J Ophthalmol 59:649

37. Waltman S, Krupin T, Hanish S, Oestrich C, Becker B (1978) Alteration of the blood-retinal barrier in experimental diabetes mellitus. Arch Ophthalmol 96: 878-879

38. Jones CW, Cunha-Vaz J, Żweig KO, Stein M (1979) Kinetic vitreous fluorophotometry in experimental diabetes. Arch Ophthalmol 97: 1941

39. Tso MOM, Cunha-Vaz J, Blair N, Jones CW, Suvaizdis I, Jonasson $\mathrm{O}$ (1982) Disruption of blood-retinal barrier in diabetic primates. ARVO abstracts. Invest Ophthalmol Vis Sci 22 [Suppl]: 110

40. Ishibachi T, Tanaka K, Taniguchi Y (1980) Disruption of bloodretinal barrier in experimental diabetic rats: an electron microscopic study. Exp Eye Res 30: 401-410

Received: 20 June 1989

and in revised form: 27 October 1989

Dr. A. Dosso

Clinique d'Ophthalmologie

Hôpital Cantonal Universitaire

22 , rue Alcide-Jentzer

CH-1211 Geneva 4

Switzerland 